\title{
PC-AR : Apoio ao Ensino de Organização de Computadores utilizando Realidade Aumentada
}

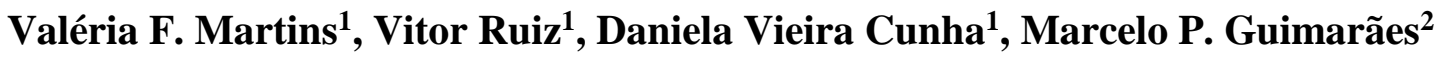 \\ ${ }^{1}$ Faculdade de Computação e Informática - Universidade Presbiteriana Mackenzie \\ Rua da Consolação, 930 Cep 01302-907 - Consolação - São Paulo - SP - Brasil \\ ${ }^{2}$ Universidade Aberta do Brasil - Universidade Federal de São Paulo (UAB/Unifesp) \\ /Programa de mestrado da Faculdade Campo Limpo Paulista, São Paulo, Brasil
}

\{valfarinazzo,vih_ruiz\}@hotmail.com, daniela.cunha@mackenzie.br, marcelodepaiva@gmail.com

\begin{abstract}
Some introductory courses in Computer Science are considered difficult for students, especially those related to hardware, such as Computer Organization. This problem motivated the development of PC-AR (Personal Computer - Augmented Reality), an Augmented Reality application that can enhance the student's perception of the selected materials in Computer Organization course. The evaluation this application using responses from 12 students and the results are presented in this work.
\end{abstract}

Resumo. Algumas disciplinas introdutórias dos cursos de Computação são consideradas difíceis para os alunos, principalmente aquelas relacionadas com hardware, como é o caso de Organização de Computadores. Isso motivou o desenvolvimento do PC-AR (Personal Computer - Augmented Reality), que é uma aplicação de Realidade Aumentada voltada para aumentar a percepção dos alunos em relação aos materiais da disciplina Organização de Computadores. Essa aplicação e os resultados da avaliação aplicada, que foi realizada por 12 alunos universitários, são apresentados nesse trabalho.

\section{Introdução}

Segundo Torres (2012), estudantes de Computação enfrentam dificuldades com disciplinas introdutórias, especialmente as ligadas a hardware, como é o caso de Organização e Arquitetura de Computadores (OAC), o que requer então metodologias, técnicas, ferramentas e aplicações que facilitem o aprendizado. Por isso, ele propões que o aprendizado desta disciplina não seja tão teórico e que seja possível ao estudante experimentar e visualizar o conhecimento acerca do funcionamento do computador. Sousa et al. (2012) também compartilham a mesma linha de argumentação, sugerindo então o uso de simuladores como ferramentas de suporte para o processo de ensinoaprendizagem.

Por outro lado, a Realidade Aumentada (RA) foi apontada pelo Horizon Report [NMC 2012] como uma das tecnologias que irão influenciar a Educação nos próximos anos. Isso se deve, entre outras razões, a seu caráter lúdico e interativo. No entanto, o uso de RA para o ensino de OAC parece ainda estar bastante insipiente, pois poucos trabalhos relacionados ao tema foram encontrados na literatura [Silva et. al 2012; Majid 2013]. 
O objetivo deste trabalho foi investigar o uso de RA para a criação de aplicações/jogos que possam contribuir para tornar a disciplina OAC menos abstrata para os estudantes. Para tanto, foi desenvolvida uma aplicação contendo jogos em RA, denominada PC-AR (Personal Computer - Augmented Reality). Além de mostrar essa aplicação, esse artigo apresenta os testes de aprendizagem realizados.

Este artigo está estruturado da seguinte maneira. A seção 2 apresenta os trabalhos relacionados. A seção 3 apresenta a aplicação PC-AR. A seção 4 apresenta a metodologia utilizada para os testes. Na seção 5 são discutidos os resultados obtidos com a aplicação dos jogos para estudantes do curso de Ciência da Computação da universidade foco deste estudo. Finalmente, na seção 6 são apresentadas as considerações deste trabalho.

\section{Trabalhos relacionados}

RA tem sido utilizada em várias áreas do conhecimento como ferramenta para auxiliar o processo de ensino-aprendizagem, como, por exemplo, na Matemática e Física [Forte 2009], Arquitetura e Construção Civil [Rodrigues, Pinto e Rodrigues, 2010] e Artes [Braga 2011]. No entanto, o uso de RA para o ensino de OAC é insipiente, com poucos trabalhos relacionados. Silva, Carvalho e Rodrigues (2012) usaram essa tecnologia para melhorar a detenção do conhecimento sobre abstração. Para tanto, exploraram o modelo de Von Neumann. Os autores mostraram como resultado a interface da aplicação, porém nenhum teste com usuários, não sendo possível, assim, concluir questões como: se a ferramenta auxilia o aprendizado, se os usuários se sentem motivados a utilizá-la, se a consideraram lúdica, entre outros pontos.

Já Majid (2013) apresenta uma aplicação de RA móvel para auxiliar no processo de ensino-aprendizagem de Organização de Computadores e Sistemas Operacionais. A aplicação foi avaliada por 13 estudantes do segundo ano universitário. Segundo o autor, a avaliação aponta que este aplicativo pode ser usado para melhorar a compreensão dos estudantes e envolvê-los em uma discussão em grupo para resolver os exercícios dados.

\section{Personal Computer - Augmented Reality (PC-AR)}

A aplicação PC-AR foi concebida, por um estudante de $1^{\circ}$ ano, que havia cursado a disciplina de Organização de Computadores. Este estudante sentiu dificuldades de visualização de componentes e de formas mais interativas para aprender a disciplina.

PC-AR foi implementada utilizando a ferramenta Flaras [Souza, Moreira e Kirner 2014]. Ela concentra-se em apresentar alguns dos principais componentes de um computador (processadores, memórias, dispositivos de entrada e saída). Em relação ao previsto pelas Diretrizes Curriculares da SBC [Diretrizes 2003], o intuito foi prover habilidades e competências aos alunos em relação a compreender os conceitos ligados ao hardware e seu funcionamento. Também foi objetivo obter o caráter lúdico dos jogos de computador e ainda atender os seguintes requisitos: ser fácil de usar, fácil de aprender, intuitiva e visualmente agradável.

A aplicação foi dividida em dois jogos que permitem a interação do usuário com a aplicação. Em ambos os jogos, no painel inicial estão distribuídas quatro peças na parte superior que, quando clicadas, emitem um breve áudio explicativo, que corresponde à parte do processo de ensino-aprendizado. Logo abaixo, encontra-se um 
campo dividido em quatro/duas áreas com uma classificação pré-definida. O objetivo dos jogos é que o usuário consiga classificar cada objeto da tela. Assim, o estudante deve arrastar as peças da parte superior à área de correspondência, que se encontra na parte inferior da tela (Figura 1).

O PC-AR alia aprendizagem a novos recursos tecnológicos, o que pode tornar todo o processo didático mais interessante. Além disso, por se tratar de uma tecnologia não convencional nos dias de hoje, a aplicação conta com instruções para auxiliar no uso da nova interface. A Figura 1 apresenta os passos de execução do primeiro jogo do PC-AR. A interface do segundo jogo é semelhante.

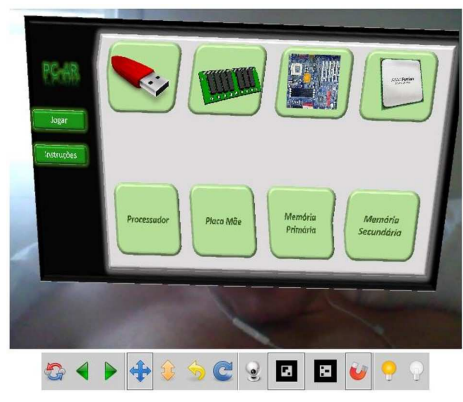

Passo 1

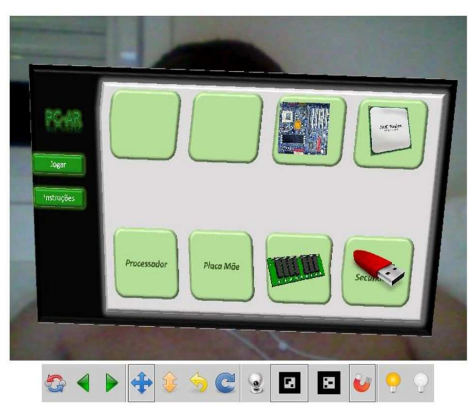

Passo 2

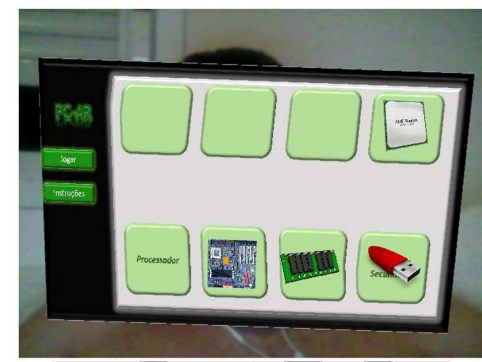

Q4D C : 回回

Passo 3

Figura 1 - Passos 1, 2 e 3 de Execução do Primeiro Jogo

\section{Metodologia}

Para validar a aplicação, foram realizados testes com um grupo de 12 estudantes do curso de Ciência da Computação. O grupo foi formado por quatro mulheres e oito homens. Parte destes estudantes já tinha cursado a disciplina de Organização de Computadores (oito pessoas). A faixa etária estava entre 17 e 20 anos. Seis estudantes já tinham tido contato com RA. Para verificar o aprendizado, foi realizado um pré-teste contendo conteúdos relacionados aos jogos e um pós-teste, em que foi verificado o ganho de aprendizado (Tabela I). O tempo médio para a execução de cada jogo foi de 5 minutos. Após a utilização dos jogos, os alunos realizaram o pós-teste (prova dos conteúdos) e responderam a um questionário de satisfação.

\section{Tabela I. Pré e pós-teste}

\section{PRÉ-TESTE/ PÓS-TESTE}

1.Explique o que você entende por memória

4.Explique quais as funções da UCP

2.Explique o que você entende por memória principal $\quad$ 5.Cite dois componentes da placa-mãe.

3.Cite dois exemplos de memória secundária

O tempo total, incluindo pré-teste, uso dos jogos e pós-teste, foi de 45 minutos, em média. Os ambientes físicos utilizados para os testes não foram ambientes controlados. Todos os testes foram realizados numa sala de aulas composta por notebooks, em horário diferente ao das aulas. 


\section{Resultados e Discussões}

Após a aplicação da Metodologia, foi possível captar alguns resultados em relação ao aprendizado da aplicação PC-AR. As notas de cada aluno antes e depois do uso dos jogos (pré-teste e pós-teste) são mostradas na Tabela II. Os alunos enumerados por 2, 4, 6 e 7 são os alunos que ainda não tiveram contato com o tema.

Tabela II. Valores dos testes

\begin{tabular}{|c|c|c|c|c|c|c|c|c|c|c|c|c|}
\hline Aluno & 1 & 2 & 3 & 4 & 5 & 6 & 7 & 8 & 9 & 10 & 11 & 12 \\
\hline Pré-teste & 6,5 & 3 & 7 & 2 & 9 & 3,5 & 1 & 6 & 6,5 & 5 & 7 & 7 \\
\hline Pós-teste & 8 & 6 & 7,5 & 7,5 & 9 & 7 & 7 & 9 & 8 & 8,5 & 9 & 9,5 \\
\hline $\begin{array}{l}\text { Ganho } \\
(\%)\end{array}$ & 23,08 & 100 & 7,14 & 275 & 0 & 100 & 600 & 50 & 23,08 & 70 & 28,57 & 35,71 \\
\hline
\end{tabular}

Através desta tabela é possível perceber que:

- Pré-teste: média de 5,2 com desvio-padrão de 2,58;

- Pós-teste: média de 8,0 com desvio-padrão de 1,04;

- Apenas um aluno manteve seu conhecimento indiferente; os demais apresentaram ganho de conhecimento;

- Os alunos que não tinham conhecimento prévio, foram os alunos que mais tiveram ganho de aprendizagem com os jogos;

- Embora parte dos alunos já tivesse tido contato com a disciplina, o pré-teste mostrou que muitos conceitos haviam sido esquecidos. Para a outra parte dos alunos que não havia cursado a disciplina, o seu nível de conhecimento sobre o assunto era bastante reduzido, muito embora todos os alunos fossem do curso de Ciência da Computação.

Já de acordo com as questões relacionadas à disciplina, foi verificado que:

- Todos consideraram válido o uso de RA para este tipo de aprendizado;

- Todos acreditam que o uso de RA pode melhorar no desenvolvimento de conhecimento nas disciplinas;

- $25 \%$ consideraram que a aplicação exigiu muito de seus conhecimentos, $58 \%$ regular e $17 \%$ consideraram pouco.

\section{Conclusões}

Este trabalho apresentou o PC-AR, que é uma ferramenta que usa RA para o auxílio do processo de ensino-aprendizagem na disciplina de Organização de Computadores. Os testes realizados com 12 estudantes demonstraram que houve um ganho significativo de conhecimento por parte dos alunos, principalmente dos alunos que nunca tinham tido contado direto com a disciplina. Além disso, o jogo demonstrou-se como um fator motivador, o que gerou demanda para o mesmo seja utilizado em outras disciplinas para a visualização de informações complexas/abstratas. 
Os resultados apresentados apontam que RA pode ser uma tecnologia a ser explorada para o desenvolvimento de ferramentas que visam auxiliar o processo de ensino-aprendizagem da disciplina Organização de Computadores. Contudo, ainda existe a necessidade de mais testes com mais estudantes e para outras disciplinas.

\section{Agradecimentos}

Os autores agradecem a CAPES pela bolsa de Iniciação Científica - PIBIC - destinada ao autor Vitor Ruiz - e também à Universidade Presbiteriana Mackenzie por ceder o espaço físico, instalações e recursos computacionais para a efetivação do projeto.

\section{Referências}

Braga, I. F.; Landau, L. and Cunha, G. G.(2011) "Realidade Aumentada em Museus: As Batalhas do Museu Nacional de Belas Artes". Virtual Reality, v. 4, n. 1, p. 35-55.

Diretrizes Curriculares da SBC (2003). Disponível em: http://www.sbc.org.br/index.php?option=com_jdownloads\&Itemid=195\&task=finish $\&$ cid $=186 \&$ catid=36. Acessado em 30 de maio de 2014 .

Forte, C. and Kirner, C. (2009) "Usando Realidade Aumentada no Desenvolvimento de Ferramenta para Aprendizagem de Física e Matemática". In: $6^{\circ}$ Workshop de Realidade Virtual e Aumentada, Santos - SP : UNISANTA, v. 1. p. 1-6.

Majid, N.A.A. (2013) "Application of Mobile Augmented Reality in a Computer Science Course". In: Advances in Visual Informatics. Springer International Publishing, p. 516-525.

NMC (2012), "NMC Horizon Project Short List: 2013 Higher Education Edition", Austin, Texas, The New Media Consortium. Available:http://www.nmc.org/pdf/2013-horizon-higher-edshortlist.pdf. Acessado em 10 de maio de 2014.

Rodrigues,C. S. C. ; Pinto, R. A. M. and Rodrigues, P. F. N. (2010) "Uma aplicação da realidade aumentada no ensino de modelagem dos sistemas estruturais". Revista Brasileira de Computação Aplicada, v. 2, n. 2, p. 81-95.

Silva, E. P.; Carvalho, A R. S. and Rodrigues, C. M. O. (2012) "Realidade Aumentada Aplicada no Ensino de Arquitetura de Computadores". In: VII CONNEPI Congresso Norte e Nordeste de Pesquisa e Inovação, 2012, Palmas. Realidade Aumentada Aplicada no Ensino de Arquitetura de Computadores.

Sousa, T.D.N.; Souza, C. C.; Silva, E. L. Silva and Azevedo, R. R. (2012) "Um Simulador para Apoiar no Processo de Ensino e Aprendizagem de Organização e Arquitetura de Computadores". Workshop sobre Educação em Computação, Curitiba. Anais do XX Workshop sobre Educação em Computação.

Souza, R. C. Moreira, H. D. F. and Kirner, C. (2014) "FLARAS 2.0-Flash Augmented Reality Authoring System", Disponível em: <http://ckirner.com/flaras/>. Acesso em: 22 maio 2014.

Torres, A.L. (2012). "Simulação baseada em atores como ferramenta de ensino de organização e arquitetura de computadores". Universidade Federal da Paraíba. Departamento de Informática. Programa de Pós-Graduação em Informática. Dissertação de Mestrado. Março. João Pessoa, PB.112 páginas. 